\section{RMD Open}

Rheumatic \&

Musculoskeletal Diseases

\title{
Predictors of remission in patients with
} non-radiographic axial spondyloarthritis receiving open-label
adalimumab in the ABILITY-3 study

Joachim Sieper, ${ }^{\oplus 1}$ Robert Landewé, ${ }^{2,3}$ Marina Magrey, ${ }^{4}$ Jaclyn K Anderson, ${ }^{\oplus}$ Sheng Zhong, ${ }^{5}$ Xin Wang, ${ }^{5}$ Apinya Lertratanakul ${ }^{5}$

To cite: Sieper J, Landewé $\mathrm{R}$, Magrey M, et al. Predictors of remission in patients with non-radiographic axial spondyloarthritis receiving open-label adalimumab in the ABILITY-3 study. RMD Open 2019;5:e000917. doi:10.1136/ rmdopen-2019-000917

- Additional material is published online only. To view please visit the journal online (http://dx.doi.org/10. 1136rmdopen-2019-000917).

This manuscript was based on part of the work previously presented at the 2017 European Congress of Rheumatology and published as a conference abstract.

Received 4 February 2019 Revised 25 April 2019 Accepted 15 May 2019

Check for updates

\section{(C) Author(s) (or their} employer(s)) 2019. Re-use permitted under CC BY-NC. No commercial re-use. See rights and permissions. Published by BMJ.

For numbered affiliations see end of article.

Correspondence to Dr Joachim Sieper; joachim.sieper@charite.de

\section{ABSTRACT}

Background This analysis assessed baseline predictors of remission in patients with non-radiographic axial spondyloarthritis (nr-axSpA) who received open-label adalimumab therapy.

Methods ABILITY- 3 enrolled 673 adult patients with nraxSpA who had objective evidence of inflammation by MRI or elevated high-sensitivity $\mathrm{C}$ reactive protein at screening, active disease and an inadequate response to two or more non-steroidal anti-inflammatory drugs. Patients received adalimumab $40 \mathrm{mg}$ every other week during a 28-week open-label lead-in period. Clinical remission was defined as Ankylosing Spondylitis Disease Activity Score inactive disease (ASDAS ID; score <1.3) and Assessment of SpondyloArthritis international Society partial remission (ASAS PR; score $<2 / 10$ in each of the four ASAS domains). Stepwise logistic regression was used to identify baseline predictors of remission at week 12 and at final visit (last postbaseline visit up to week 28). Only patients without missing data were included.

Results Overall, 593 patients were included in the ASDAS ID and 596 in the ASAS PR analysis at week 12. Younger age ( $\leq 45$ years), male sex, positive human leucocyte antigen (HLA)-B27 and higher Spondyloarthritis Research Consortium of Canada (SPARCC) MRI sacroiliac joint score were consistent predictors of remission by both ASAS ID and ASDAS PR at week 12. Results were generally similar in the final visit analysis. Other variables did not consistently predict remission.

Conclusions In ABILITY-3, consistent and strong baseline predictors of remission included younger age, male sex, HLA-B27 positivity and higher SPARCC MRI sacroiliac joint score among patients with active nr-axSpA receiving adalimumab therapy, similar to previous findings in ankylosing spondylitis.

\section{INTRODUCTION}

Patients with active non-radiographic axial spondyloarthritis (nr-axSpA) are candidates for tumour necrosis factor (TNF) inhibitor therapy if they have clinically active disease despite treatment with non-steroidal anti-inflammatory drugs (NSAIDs) and objective evidence of

\section{Key messages}

What is already known about this subject?

- Patients with non-radiographic axial spondyloarthritis (nr-axSpA) are candidates for tumour necrosis factor (TNF) inhibitor therapy if they have clinically active disease despite non-steroidal anti-inflammatory drugs and objective evidence of inflammation by either elevated $\mathrm{C}$ reactive protein and/or definite inflammation on MRI.

- In patients with ankylosing spondylitis, shorter disease duration, younger age, male sex and presence of human leucocyte antigen (HLA)-B27 have been identified as potential predictors of clinical response to TNF inhibitor therapy; however, less is known about predictors of remission with TNF inhibitor therapy in patients with nr-axSpA.

What does this study add?

- Baseline predictors of remission after open-label adalimumab therapy were identified using data from ABILITY-3, the largest clinical trial to date of patients with active $\mathrm{nr}-\mathrm{axSpA}$.

- Younger age ( $\leq 45$ years), male sex, HLA-B27 positivity and higher Spondyloarthritis Research Consortium of Canada (SPARCC) MRI sacroiliac joint score were consistent predictors of clinical remission (and clinical response) at week 12 in patients with active $n r-a x S p A$ receiving adalimumab therapy, with similar findings observed at last postbaseline visit up to week 28.

How might this impact on clinical practice?

- Remission is a sought-after treatment goal for patients with axSpA, and identification of baseline predictors of remission with adalimumab treatment may aid in clinical management of the disease.

inflammation by elevated $\mathrm{C}$ reactive protein (CRP) and/or MRI. ${ }^{1}$ Previous studies have identified predictors of clinical response to TNF inhibitor therapy in patients with ankylosing spondylitis (AS), including shorter 
disease duration, younger age, elevated CRP, male sex and presence of human leucocyte antigen (HLA)-B2 $7^{2-8}$; however, none of these factors is considered adequate alone or in combination to select patients for treatment. In addition, less is known about predictors of remission in patients with nr-axSpA. ${ }^{9-11}$

Adalimumab, a TNF inhibitor, is indicated for the treatment of adult patients with nr-axSpA. ${ }^{12}$ The ABILITY-3 (NCT01808118) study assessed whether adalimumab can be discontinued in patients with nr-axSpA who are in sustained remission after 28 weeks of open-label adalimumab treatment. ${ }^{13}$ During the open-label period, $59 \%$ (373/637) of patients achieved $40 \%$ improvement in Assessment of SpondyloArthritis international Society (ASAS40), 35\% (220/633) of patients achieved Ankylosing Spondylitis Disease Activity Score inactive disease (ASDAS ID) and 22\% (138/636) achieved ASAS partial remission (ASAS PR) at week 12, and $71 \%(478 / 670)$, $54 \%(361 / 668)$ and $41 \%(272 / 670)$ of patients, respectively, achieved these at their final open-label period visit (last postbaseline visit at up to 28 weeks of treatment). At the end of the 28 -week open-label period, $45 \%$ of patients achieved sustained remission and were randomised to continuous adalimumab or withdrawal of adalimumab (placebo) during a 40-week double-blind period. Lower ASDAS (range 0.0-1.3) at week 28 was the only consistent predictor of drug-free remission and remission maintenance with continued adalimumab therapy through week 68; however, the validity of ASDAS values below the cut-off for inactive disease is undefined. ${ }^{13}$

Remission is a desirable treatment goal for patients with axSpA, ${ }^{14}$ and thus identification of baseline predictors of remission with TNF inhibitor therapy, especially at 12 weeks, which is the recommended first treatment re-evaluation time point, ${ }^{1}$ may further aid in clinical management of nr-axSpA. The objective of this analysis was to assess baseline predictors of remission in patients with nr-axSpA at week 12 and up to week 28 of open-label adalimumab therapy in the ABILITY-3 study.

\section{METHODS}

\section{Patients}

The methods and efficacy and safety outcomes for the open-label and double-blind periods of ABILITY-3 have been reported previously. ${ }^{13}$ Briefly, ABILITY-3 enrolled adult patients with nr-axSpA who fulfilled the ASAS criteria but not the radiologic criterion of the modified New York criteria for AS. To be eligible, patients also had to have objective evidence of active inflammation in the sacroiliac (SI) joints or spine on MRI or elevated high-sensitivity $\mathrm{C}$ reactive protein (hs-CRP, that is, greater than upper limit of normal for the laboratory) at screening, active disease at baseline (defined as ASDAS 22.1, Bath Ankylosing Spondylitis Disease Activity Index (BASDAI) $\geq 4$ and patient assessment of total back pain score $\geq 4$ based on a numeric rating scale of 0 to 10) and an inadequate response to at least two NSAIDs, intolerance to NSAIDs or contraindication for NSAIDs.

\section{Study design}

ABILITY-3 included a 28-week open-label lead-in period, followed by a 40-week double-blind randomised period. During the open-label lead-in period, all patients received open-label adalimumab $40 \mathrm{mg}$ every other week for 28 weeks. Patients who achieved sustained remission during the open-label period, defined as achieving ASDAS ID (ASDAS score <1.3) at weeks 16, 20, 24 and 28, were randomised to placebo or adalimumab for an additional 40 weeks during double-blind period 2; patients who did not achieve sustained remission during the open-label period were discontinued from the study starting at week 20. Data from the 28-week open-label lead-in period were included in this analysis.

\section{Predictor analysis}

Stepwise logistic regression was used to identify potential baseline predictors of remission at week 12 during the open-label lead-in period 1. Additionally, predictors at final visit were assessed (online supplementary file). The final visit was determined as the last postbaseline visit that occurred during the open-label period, corresponding to the week 28 visit for patients who were randomised into period 2 and the last open-label visit (either at week 2, 4, $8,12,16,20,24$ or 28 ) for patients who discontinued the study during the open-label period 1. Clinical remission was defined as ASDAS ID (ASDAS score $<1.3$ ) or ASAS PR (absolute score $<2$ on a scale of 0 to 10 for each of the following domains: Patient Global Assessment of Disease Activity (PtGA), patient assessment of total back pain, Bath Ankylosing Spondylitis Functional Index (BASFI) and morning stiffness as a measure of inflammation (mean of items 5 and 6 of the BASDAI)). A total of four models were assessed: ASDAS ID at week 12 and final visit and ASAS PR at week 12 and final visit. Additionally, predictors of ASDAS Major Improvement (reduction from baseline $\geq 2.00$ ) and ASAS 40 response (defined as improvement of $\geq 40 \%$ and absolute improvement of $\geq 2$ units on a scale of 0 to 10 from baseline in $\geq 3$ of the 4 domains above with no deterioration in the potential remaining domain) were analysed. The stepwise regression analysis was based on observed data, where patients with missing predictor or response values were not included. Significant predictors $(\mathrm{p}<0.05)$ and predictors with strong statistical significance $(p \leq 0.005)$ for an association with achievement of remission were identified.

The following continuous baseline variables were included in the model: age, symptom duration, disease duration, BASDAI, ASDAS, BASFI, Physician Global Assessment of disease activity, PtGA, Health Assessment Questionnaire modified for the Spondyloarthropathies (HAQ-S), hs-CRP, morning stiffness, patient assessment of total back pain, Spondyloarthritis Research Consortium of Canada (SPARCC) MRI SI joint score, and SPARCC MRI spine score. 
The following categorical baseline variables were included in the model: sex (male vs female), age $>35$ years, age $>45$ years, hs-CRP greater than upper limit of normal $(2.87 \mathrm{mg} / \mathrm{L})$, presence of inflammation on MRI of the SI joints and presence of inflammation on MRI of the SI joints and/or spine (as single variable) by centrally read MRI, the presence of HLA-B27, and concomitant use of conventional synthetic disease-modifying antirheumatic drugs (DMARDs), NSAIDs and corticosteroids.

\section{RESULTS}

A total of 673 patients were enrolled in ABILITY-3. Final study visit in the open-label period was most commonly at week $28(325(48 \%))$ or week $20(276(41 \%))$, with the remainder of the patients discontinuing at week 24 $(20(3 \%))$ or at $\leq 16$ weeks $(52(8 \%))$. Among patients without missing data, $593(88 \%)$ patients were included in the ASDAS ID predictor analysis and 596 (89\%) were included in the ASAS PR predictor analysis at week 12. Similarly, $654(97 \%)$ and $656(97 \%)$ patients were included in the ASDAS ID and ASAS PR predictor analyses at final visit, respectively (online supplementary file).

Most baseline characteristics were significantly different between week 12 ASDAS ID and ASAS PR responders versus non-responders (table 1). Responders were significantly younger, had shorter symptom duration, were more likely to be male and HLA-B27 positive, and had lower total back pain, BASDAI, PtGA, BASFI and HAQ-S scores and higher SPARCC MRI SI joint at baseline versus non-responders. Mean hs-CRP levels at baseline were numerically higher in ASDAS ID non-responders versus ASDAS ID responders (10.9 vs 9.1) and a significantly greater proportion of ASDAS ID non-responders had elevated hs-CRP at baseline $(68 \%$ vs $56 \%(\mathrm{p}=0.0065))$ versus ASDAS ID responders. Interestingly, among ASAS PR responders, mean hs-CRP level at baseline was nearly twice than that of non-responders (15.6 vs 8.8; $\mathrm{p}<0.0001$ ); however, the proportion of patients with elevated hs-CRP at baseline was similar between responders and non-responders (67\% and 63\%).

In the final visit analysis, baseline characteristics between ASDAS ID and ASAS PR responders and non-responders were generally different as in the week 12 analysis (online supplementary table 1 ).

\section{Baseline predictors of ASDAS ID and ASAS PR}

Achievement of ASDAS ID at week 12 was associated with lower ASDAS, lower BASFI, more severe morning stiffness, higher SPARCC MRI SI joint score, younger age ( $\leq 45$ years), male sex and presence of HLA-B27 at baseline ( $\mathrm{p} \leq 0.005$, figure 1$)$. Lower SPARCG MRI spine score at baseline also predicted ASDAS ID at week 12 $(\mathrm{p}=0.014)$.

Lower HAQ-S, higher hs-CRP level, higher SPARCC MRI SI joint score, younger age ( $\leq 45$ years) and male sex at baseline showed strong statistical significance $(p \leq 0.005)$ for an association with achievement of ASAS
PR at week 12 (figure 1). Presence of HLA-B27 was also a predictor of ASAS PR at week $12(\mathrm{p}=0.009)$.

Consistent with the week 12 analysis, younger age (continuous variable), male sex and positive HLA-B27 were also found to be predictors of ASDAS ID and ASAS PR remission in the final visit analysis (online supplementary figure 1). Additionally, presence of or higher SI joint inflammation on MRI, lower total back pain and normal hs-CRP were strong predictors of ASDAS ID and/ or ASAS PR, whereas higher morning stiffness and use of concomitant DMARDs or NSAIDs marginally predicted remission in the final visit analysis.

\section{Baseline predictors of ASDAS Major Improvement and ASAS40}

Higher ASDAS, lower BASFI, younger age, male sex, presence of HLA-B27 and higher SPARCC MRI SI joint score at baseline showed strong statistical significance $(\mathrm{p} \leq 0.005)$ for an association with achievement of ASDAS Major Improvement at week 12 (figure 2).

Achievement of ASAS40 at week 12 was associated with more severe morning stiffness, male sex and higher hs-CRP ( $\mathrm{p} \leq 0.005$; figure 2 ). Younger age ( $\leq 45$ years), lower HAQ-S and higher SPARCC MRI SI joint score at baseline also predicted ASAS40 at week $12(p<0.05)$.

\section{DISCUSSION}

This analysis of data from the ABILITY-3 study demonstrated that in patients with nr-axSpA younger age $(\leq 45$ years), male sex, positive HLA-B27 and higher SPARCC MRI SI joint score at baseline were consistent predictors of remission by both ASDAS ID and ASAS PR at week 12 . Similar results were observed at the final visit analysis, with the exception that higher SPARCC MRI SI joint score was not a significant predictor of ASDAS ID, while presence of SI joint inflammation on MRI was. Higher morning stiffness was also a predictor of both ASDAS ID and ASAS PR in three of the four models used (with the exception of ASAS PR at week 12). Other statistically strong baseline predictors of ASDAS ID $(\mathrm{p} \leq 0.005)$ included lower disease activity (ASDAS) and better functional status (lower BASFI) at week 12. Less inflammation on MRI of the spine (lower SPARCC MRI spine score) at baseline marginally predicted ASDAS ID at week 12, although this is probably of low relevance for $\mathrm{nr}-\mathrm{axSpA}$ as reflected in the low baseline values compared with SPARCC MRI SI joint scores and the small difference shown between responders and non-responders. For ASAS PR, better functional status (lower HAQ-S) and higher hs-CRP at baseline were strong predictors $(\mathrm{p} \leq 0.005)$ at week 12 .

We also assessed the predictors of ASDAS Major Improvement and ASAS40 at week 12. The results were generally consistent with the predictors of remission and demonstrated that younger age, male sex and higher SPARCC MRI SI joint score at baseline were significant predictors of both ASDAS Major Improvement and ASAS40. Positive HLA-B27 was a predictor of ASDAS 
Table 1 Baseline characteristics of ASDAS ID and ASAS PR responders and non-responders at week 12 of open-label adalimumab treatment period

\begin{tabular}{|c|c|c|c|c|}
\hline \multirow[b]{2}{*}{ Characteristic } & \multicolumn{2}{|l|}{ ASDAS ID } & \multicolumn{2}{|l|}{ ASAS PR } \\
\hline & $\begin{array}{l}\text { Responders } \\
(\mathrm{n}=209)\end{array}$ & $\begin{array}{l}\text { Non-responders } \\
(\mathrm{n}=384)\end{array}$ & Responders (n=130) & $\begin{array}{l}\text { Non-responders } \\
(n=466)\end{array}$ \\
\hline Age (years) & $33.7 \pm 9.8$ & $38.9 \pm 11.4^{\star \star \star}$ & $32.0 \pm 8.7$ & $38.5 \pm 11.3^{\star \star \star}$ \\
\hline Male, n (\%) & $136(65)$ & $156(41)^{\star \star \star}$ & $89(68)$ & $206(44)^{\star \star \star}$ \\
\hline Age $>35$ years, $n(\%)$ & $79(38)$ & $215(56)^{\star \star \star}$ & $43(33)$ & $252(54)^{\star \star \star}$ \\
\hline Age $>45$ years, $n(\%)$ & $23(11)$ & $113(29)^{\star \star \star}$ & $7(5)$ & $129(28)^{\star \star \star}$ \\
\hline Diagnosis duration, years & $1.7 \pm 2.9$ & $1.8 \pm 3.6$ & $1.7 \pm 3.0$ & $1.8 \pm 3.2$ \\
\hline Symptom duration, years & $6.1 \pm 6.2$ & $8.3 \pm 8.1^{\star \star \star}$ & $5.3 \pm 5.7$ & $8.0 \pm 7.8^{\star \star \star}$ \\
\hline HLA-B27 positive, n (\%) & $181(87)$ & $271(71)^{\star \star \star}$ & $116(89)$ & $338(73)^{\star \star \star}$ \\
\hline $\begin{array}{l}\text { Concomitant DMARDs at } \\
\text { baseline, } \mathrm{n}(\%)\end{array}$ & $46(22)$ & $78(20)$ & $33(25)$ & $91(20)$ \\
\hline $\begin{array}{l}\text { Concomitant NSAIDs at baseline, } \\
\mathrm{n}(\%)\end{array}$ & $157(75)$ & $271(71)$ & $106(82)$ & $325(70)^{\star \star}$ \\
\hline $\begin{array}{l}\text { Concomitant corticosteroids at } \\
\text { baseline, } \mathrm{n}(\%)\end{array}$ & $20(10)$ & $54(14)$ & $11(8)$ & $63(14)$ \\
\hline ASDAS & $3.4 \pm 0.8$ & $3.7 \pm 0.8^{\star * *}$ & $3.7 \pm 0.9$ & $3.6 \pm 0.8$ \\
\hline BASDAI & $6.6 \pm 1.3$ & $7.1 \pm 1.4^{\star \star \star}$ & $6.6 \pm 1.4$ & $7.0 \pm 1.4^{\star \star}$ \\
\hline Morning stiffness $†$ & $6.9 \pm 1.8$ & $7.0 \pm 2.0$ & $6.9 \pm 1.9$ & $7.0 \pm 1.9$ \\
\hline PGA & $6.3 \pm 1.5$ & $6.7 \pm 1.5^{\star \star}$ & $6.4 \pm 1.5$ & $6.6 \pm 1.5$ \\
\hline PtGA & $7.1 \pm 1.7$ & $7.6 \pm 1.6^{\star \star \star}$ & $7.1 \pm 1.7$ & $7.5 \pm 1.6^{\star}$ \\
\hline Total back pain & $7.0 \pm 1.7$ & $7.7 \pm 1.5^{\star \star \star}$ & $7.1 \pm 1.8$ & $7.5 \pm 1.6^{\star \star}$ \\
\hline hs-CRP & $9.1 \pm 13.1$ & $10.9 \pm 16.8$ & $15.6 \pm 21.5$ & $8.8 \pm 13.2^{\star \star \star}$ \\
\hline Elevated hs-CRP (>ULN)‡, n (\%) & $118(56)$ & $260(68)^{\star \star}$ & $87(67)$ & $293(63)$ \\
\hline BASFI & $4.6 \pm 2.2$ & $5.7 \pm 2.2^{\star \star \star}$ & $4.9 \pm 2.4$ & $5.4 \pm 2.2^{*}$ \\
\hline HAQ-S & $1.0 \pm 0.5$ & $1.2 \pm 0.6^{\star \star \star}$ & $1.0 \pm 0.6$ & $1.2 \pm 0.6^{\star \star \star}$ \\
\hline SPARCC MRI SI joint score & $10.8 \pm 13.8$ & $6.6 \pm 9.9^{* \star *}$ & $13.9 \pm 15.8$ & $6.6 \pm 9.7^{\star \star \star}$ \\
\hline SPARCC MRI spine score & $3.0 \pm 7.5$ & $4.2 \pm 8.4$ & $4.0 \pm 7.7$ & $3.8 \pm 8.2$ \\
\hline MRI SI joint inflammation, n (\%) & $164(78)$ & $260(68)^{\star \star}$ & $101(78)$ & $326(70)$ \\
\hline $\begin{array}{l}\text { MRI spine and/or SI joint } \\
\text { inflammation, } n(\%)\end{array}$ & $179(86)$ & $291(76)^{\star \star}$ & $109(84)$ & $363(78)$ \\
\hline
\end{tabular}

Data are mean \pm SD unless otherwise indicated. Statistical significance for responders versus non-responders was assessed using $t$ test for continuous variables and $\chi^{2}$ test for categorical variables.

${ }^{*}$ Indicates statistical significance at the 0.05 level, ${ }^{* *}$ Indicates statistical significance at the 0.01 level, ${ }^{* *}$ Indicates statistical significance at the 0.001 level.

†Mean of BASDAl questions 5 and 6.

†Elevated hs-CRP defined as $>2.87 \mathrm{mg} / \mathrm{L}$.

ASAS PR, Assessment of SpondyloArthritis international Society partial remission; ASDAS, Ankylosing Spondylitis Disease Activity Score; ASDAS ID, ASDAS inactive disease; BASDAI, Bath Ankylosing Spondylitis Disease Activity Index; BASFI, Bath Ankylosing Spondylitis Functional Index; DMARD, disease-modifying antirheumatic drug; hs-CRP, high-sensitivity C reactive protein; HAQ-S, Health Assessment Questionnaire modified for the Spondyloarthropathies; HLA-B27, human leucocyte antigen B27; NSAID, non-steroidal anti-inflammatory drug; PGA, Physician Global Assessment of disease activity; PtGA, Patient Global Assessment of disease activity; SI, sacroiliac; SPARCC, Spondyloarthritis Research Consortium of Canada; ULN, upper limit of normal.

Major Improvement only. High baseline ASDAS was predictive of ASDAS Major Improvement at week 12, while low baseline ASDAS was predictive of ASDAS ID at week 12. Similar to ASAS PR, better functional status (lower HAQ-S) and higher hs-CRP at baseline were also predictors of ASAS40 at week 12.

Overall, these findings are similar to previous studies assessing predictors of clinical response to TNF inhibitor therapy in patients with AS. In previous AS studies, younger age, male sex, presence of HLA-B27, elevated CRP, better physical function (measured by BASFI) and active inflammation on MRI were predictors of good clinical response (defined as $\geq 50 \%$ improvement in BASDAI50, ASAS40 or ASAS PR at week 12) to TNF inhibitor therapy. ${ }^{2-7} \mathrm{~A}$ few studies have also assessed TNF inhibitor response in patients with nr-axSpA. In 
A

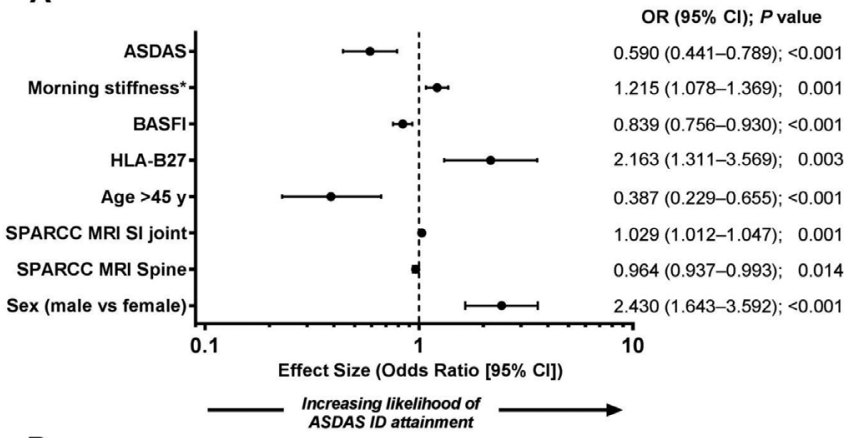

B

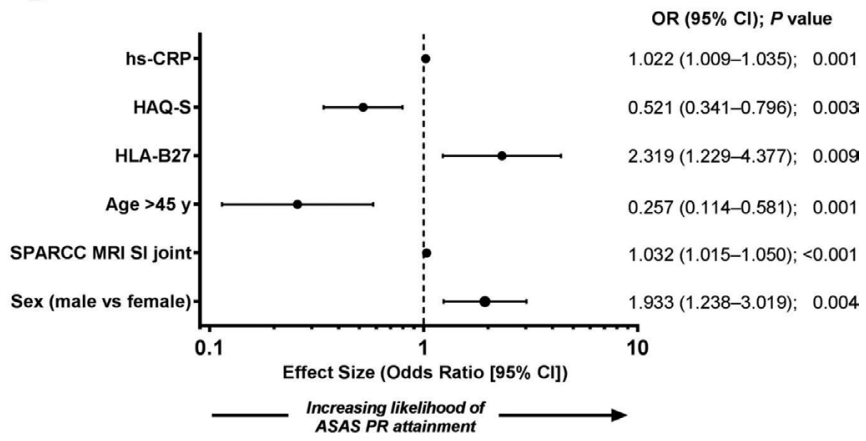

Figure 1 Baseline predictors of (A) ASDAS ID and (B) ASAS PR at week 12 of open-label adalimumab therapy. ASAS PR, Assessment of SpondyloArthritis international Society partial remission; ASDAS, Ankylosing Spondylitis Disease Activity Score; ASDAS ID, ASDAS inactive disease; BASFI, Bath Ankylosing Spondylitis Functional Index; HAQ-S, Health Assessment Questionnaire modified for the Spondyloarthropathies; HLA-B27, human leucocyte antigen B27; hs-CRP, high-sensitivity C reactive protein; SI, sacroiliac; SPARCC, Spondyloarthritis Research Consortium of Canada. *Mean of BASDAl questions 5 and 6.

the randomised, placebo-controlled ABILITY-1 study, increased baseline inflammation on MRI of the SI joints (as assessed by SPARCC score), age $<40$ years, shorter symptom duration $(<5$ years), presence of HLA-B27 and elevated CRP at baseline were associated with greater likelihood of response to adalimumab therapy in patients with nr-axSpA as measured by ASAS40 response at week $12 .{ }^{9}$ Similarly, a combination of positive MRI sacroiliitis and elevated CRP at baseline predicted response to etanercept treatment as measured by ASAS40 or BASDAI50 response at week 12 in a randomised, placebo-controlled study of patients with nr-axSpA ${ }^{10}$ and elevated CRP and/ or positive MRI inflammation in the SI joints predicted ASAS20 response in patients with nr-axSpA treated with golimumab. ${ }^{11}$ In our analysis, which assessed the predictors of remission using ASDAS ID and ASAS PR, higher SPARCC SI joint score (more SI joint inflammation) was a predictor of remission at both week 12 and at final visit and presence of SI joint inflammation on MRI was a predictor of remission by ASDAS ID at final visit. Higher SPARCC SI joint score was also a predictor of ASDAS Major Improvement and ASAS40 at week 12. Similar to the ABILITY-1 study results, ${ }^{9}$ this finding may suggest
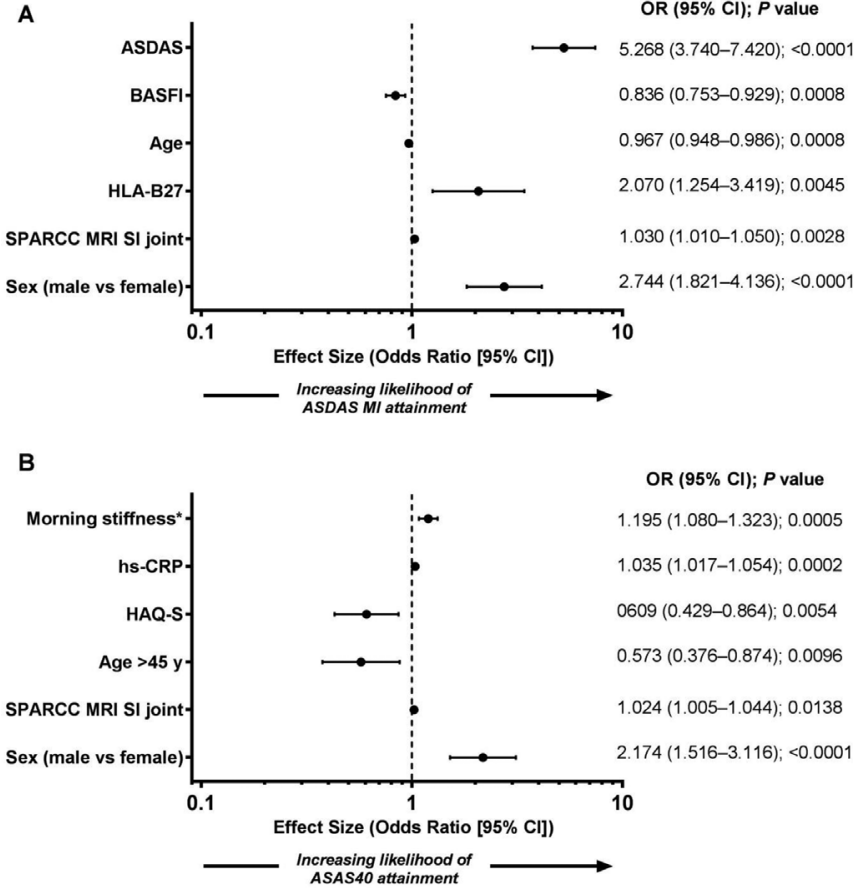

Figure 2 Baseline predictors of (A) ASDAS Major Improvement and (B) ASAS40 at week 12 of open-label adalimumab therapy. ASDAS, Ankylosing Spondylitis Disease Activity Score; ASAS40, 40\% improvement in Assessment of SpondyloArthritis international Society; BASFI, Bath Ankylosing Spondylitis Functional Index; HAQ-S, Health Assessment Questionnaire modified for the Spondyloarthropathies; HLA-B27, human leucocyte antigen B27; hs-CRP, high-sensitivity $\mathrm{C}$ reactive protein; SI, sacroiliac; SPARCC, Spondyloarthritis Research Consortium of Canada. *Mean of BASDAl questions 5 and 6.

that a greater amount of SI joint inflammation at baseline corresponds to a greater likelihood of response and remission.

Interestingly, normal hs-CRP and lower ASDAS were predictors of ASDAS ID but not ASAS PR. Previous prediction analyses in axSpA have mostly not yet used ASDAS ID as the outcome parameter but rather BASDAI50, ASAS40 and ASAS PR, ${ }^{2-10}$ of which the two latter parameters were also used in our study. ASDAS is a composite score that includes CRP, which has a relative strong weight on the score; for patients with an elevated CRP or higher ASDAS at baseline, it is more difficult to reach an ASDAS ID status compared with patients with a normal CRP or low ASDAS. This was indeed confirmed by our additional analysis of measures of change, in which higher ASDAS was a predictor of ASDAS Major Improvement and elevated CRP was a predictor of ASAS40. However, the evidence suggests that patients with symptoms such as morning stiffness and pain respond better if these symptoms are associated with objective signs of inflammation, such as elevated CRP and MRI inflammation in the SI joints, as shown in the current study.

When looking at all four models (week 12 and final visit) of remission, younger age, male sex and positive HLA-B27 were consistent predictors of remission and 
more severe morning stiffness and higher SPARCC MRI SI joint score predicted remission in three of the four models. Similar results were observed for predictors of ASDAS Major Improvement and ASAS40. These are also consistent with predictors of response to TNF inhibitor therapy in AS, as measured by ASAS PR, ASAS40 or BASDAI50 at week $12^{2-7}$ and may suggest a prominent set of predictors of response in axSpA, regardless of whether radiologic criteria for AS are fulfilled or not. However, whereas lower disease activity (ASDAS) and better functional status (BASFI or HAQ-S) predicted clinical remission at week 12 in our analysis, they were not predictors of remission at final visit. In addition, concomitant DMARDs or NSAIDs at baseline and lower total back pain predicted clinical remission at final visit but not at week 12.

This is the largest group of patients with nr-axSpA included in a predictor analysis to date. Other strengths of this analysis include that predictors were assessed at two time points using four different outcome parameters (ASDAS ID, ASAS PR, ASDAS Major Improvement and ASAS40). We focused on the analysis of predictors of remission, which has been defined as the primary treatto-target goal for axSpA. ${ }^{14}$ This differs from predictors of clinical response that were mostly analysed in other AS and nr-axSpA studies. We also included MRI inflammation as a predictor and our results were similar compared with AS studies. However, this analysis is not without limitations, including the open-label study design, although the results presented here may reflect daily clinical practice. The ABILITY-3 clinical trial was not designed to study the predictors of response to adalimumab in nr-axSpA but response to adalimumab. Only patients with objective evidence of active inflammation on MRI or by elevated hs-CRP and active disease were enrolled, which reflects the current label for starting treatment with a TNF inhibitor in the European Union and many other countries worldwide. Importantly, although we identified statistically significant baseline predictors of remission, an interpretation of these statistical results as clinically meaningful is not clear and an application to clinical practice should be approached with caution because the differences between responders and non-responders are relatively small.

Those with positive predictors and thus a statistically greater likelihood of reaching remission at week 12 should not be interpreted as an implication that those without would not benefit from therapy. In ABILITY-3, most discontinuations were forced because of failure to achieve sustained ASDAS ID; patients who did not achieve sustained remission (ASDAS ID $<1.3$ at weeks 16, 20, 24 and 28) were discontinued starting at week 20, even if they may have been improving and could have potentially reached a state of sustained remission later if permitted to stay in the study. Although reaching remission is an attractive goal, patients who show a substantial improvement in disease activity, such as reaching ASAS40, do also benefit from treatment and in clinical practice, patients who are improving (but not reaching remission yet) would generally continue treatment. Allowing patients with nr-axSpA a sufficient trial of a biological DMARD is important due to the limited therapeutic choices available.

\section{CONCLUSIONS}

In this analysis of the open-label period data from the ABILITY-3 study, HLA-B27 positivity, male sex, younger age and presence of or higher SI joint inflammation on MRI consistently predicted remission, as measured by ASDAS ID and ASAS PR, at week 12 and final visit, and mostly predicted ASDAS Major Improvement and ASAS40 (with the exception of HLA-B27 which was not a predictor of ASAS40) at week 12. Other selected variables predicted clinical remission with adalimumab therapy less consistently. Caution is warranted when interpreting these results, and future research examining combinations of outcome parameters may provide further insight into reliable predictors of remission in patients with active nr-axSpA.

\section{Author affiliations}

${ }^{1}$ Department of of Gastroenterology, Infectious Diseases, and Rheumatology, Charite Universitatsmedizin Berlin, Berlin, Germany

${ }^{2}$ Rheumatology \& Clinical Immunology, Academic Medical Center, University of Amsterdam, Amsterdam, The Netherlands

${ }^{3}$ Department of Rheumatology, Zuyderland Medical Center, Heerlen, The Netherlands

${ }^{4}$ Case Western Reserve University School of Medicine at MetroHealth Medical Center, Cleveland, Ohio, USA

${ }^{5}$ AbbVie Inc, North Chicago, Illinois, USA

Acknowledgements AbbVie for funded this study; contributed to its design; was involved in the collection, analysis and interpretation of the data; and in the writing, review and approval of the publication. Medical writing support was provided by Maria Hovenden, PhD, Complete Publication Solutions (North Wales, Pennsylvania) and was funded by AbbVie.

Contributors Statistical analysis was performed by SZ. All authors participated in study design, had access to the data, commented on the report drafts and approved the final submitted version.

Funding This study was supported by AbbVie.

Competing interests JS has received consulting fees from AbbVie, Boehringer Ingelheim, Janssen, Lilly, Merck, Novartis, Pfizer, Sun Pharma, and UCB; and speaker fees from AbbVie, Janssen, Lilly, Merck, Novartis, Pfizer and UCB. RL has received consulting or advisory board fees from Abbott/AbbVie, Ablynx, Amgen, AstraZeneca, Bristol Myers Squibb, Celgene, Janssen, Galapagos, GlaxoSmithKline, Novartis, Novo Nordisk, Merck, Pfizer, Roche, Schering-Plough, TiGenix, UCB and Wyeth; research grants from Abbott, Amgen, Centocor, Novartis, Pfizer, Roche, Schering-Plough, UCB and Wyeth; speaker fees from Abbott/AbbVie, Amgen, Bristol Myers Squibb, Janssen, Merck, Pfizer, Roche, Schering-Plough, UCB and Wyeth; and is director of Rheumatology Consultancy BV, a registered Dutch company. MM has received research grants from Amgen, AbbVie and UCB Pharma and consulting fees from Novartis and Eli Lilly. SZ, XW, AL and JKA are full-time employees of AbbVie and may own AbbVie stock and/or stock options.

Patient consent for publication Not required.

Ethics approval The study protocol was approved by an institutional review board or independent ethics committee at each study site. The study was conducted in accordance with applicable regulations and the ethical principles of Good Clinical Practice as defined by the International Conference on Harmonisation and Declaration of Helsinki. This information was included in the primary study publication (Landewé et al, Lancet 2018).

Provenance and peer review Not commissioned; externally peer reviewed.

Data availability statement This clinical trial data can be requested by any qualified researchers who engage in rigorous, independent scientific research, and will be provided following review and approval of a research proposal and 
Statistical Analysis Plan (SAP) and execution of a Data Sharing Agreement (DSA). Data requests can be submitted at any time and the data will be accessible for 12 months, with possible extensions considered. For more information on the process, or to submit a request, visit the following link: https://www.abbvie.com/ our-science/clinical-trials/clinical-trials-data-and-information-sharing/data-andinformation-sharing-with-qualified-researchers.html.

Open access This is an open access article distributed in accordance with the Creative Commons Attribution Non Commercial (CC BY-NC 4.0) license, which permits others to distribute, remix, adapt, build upon this work non-commercially, and license their derivative works on different terms, provided the original work is properly cited, appropriate credit is given, any changes made indicated, and the use is non-commercial. See: http://creativecommons.org/licenses/by-nc/4.0/.

\section{REFERENCES}

1. van der Heijde D, Ramiro S, Landewé R, et al. 2016 update of the ASAS-EULAR management recommendations for axial spondyloarthritis. Ann Rheum Dis 2017;76:978-91.

2. Rudwaleit M, Listing J, Brandt J, et al. Prediction of a major clinical response (BASDAI 50) to tumour necrosis factor alpha blockers in ankylosing spondylitis. Ann Rheum Dis 2004;63:665-70.

3. Rudwaleit M, Schwarzlose S, Hilgert ES, et al. MRI in predicting a major clinical response to anti-tumour necrosis factor treatment in ankylosing spondylitis. Ann Rheum Dis 2008;67:1276-81.

4. Rudwaleit M, Claudepierre P, Wordsworth P, et al. Effectiveness, safety, and predictors of good clinical response in 1250 patients treated with adalimumab for active ankylosing spondylitis. $J$ Rheumatol 2009;36:801-8.

5. Glintborg B, Ostergaard M, Krogh NS, et al. Predictors of treatment response and drug continuation in 842 patients with ankylosing spondylitis treated with anti-tumour necrosis factor: results from 8 years' surveillance in the Danish nationwide DANBIO registry. Ann Rheum Dis 2010;69:2002-8.
6. Arends S, Brouwer E, van der Veer E, et al. Baseline predictors of response and discontinuation of tumor necrosis factor-alpha blocking therapy in ankylosing spondylitis: a prospective longitudinal observational cohort study. Arthritis Res Ther 2011;13.

7. Maneiro JR, Souto A, Salgado E, et al. Predictors of response to TNF antagonists in patients with ankylosing spondylitis and psoriatic arthritis: systematic review and meta-analysis. RMD Open 2015;1:e000017.

8. Vastesaeger N, van der Heijde D, Inman RD, et al. Predicting the outcome of ankylosing spondylitis therapy. Ann Rheum Dis 2011;70:973-81.

9. Sieper J, van der Heijde D, Dougados M, et al. Efficacy and safety of adalimumab in patients with non-radiographic axial spondyloarthritis: results of a randomised placebo-controlled trial (ABILITY-1). Ann Rheum Dis 2013;72:815-22.

10. Brown MA, Bird PA, Robinson PC, et al. Baseline MRI and CRP as predictors of response to etanercept in the management of patients with non-radiographic axial spondyloarthritis (nr-axSpA). Arthritis Rheumatol 2015;67.

11. Sieper J, van der Heijde D, Dougados $M$, et al. A randomized, double-blind, placebo-controlled, sixteen-week study of subcutaneous golimumab in patients with active nonradiographic axial spondyloarthritis. Arthritis Rheumatol 2015;67:2702-12.

12. Humira (adalimumab). Summary of product characteristics. Maidenhead, UK: AbbVie Ltd, 2016.

13. Landewé R, Sieper J, Mease P, et al. Efficacy and safety of continuing versus withdrawing adalimumab therapy in maintaining remission in patients with non-radiographic axial spondyloarthritis (ABILITY-3): a multicentre, randomised, double-blind study. Lancet 2018;392:134-44.

14. Smolen JS, Schöls M, Braun J, et al. Treating axial spondyloarthritis and peripheral spondyloarthritis, especially psoriatic arthritis, to target: 2017 update of recommendations by an international Task Force. Ann Rheum Dis 2018;77:3-17. 